\title{
Thrombocytopenia in critically ill patients: prognostic marker in postoperative ICU
}

\author{
Domingos Dias Cicarelli*, Victor Dantas, Luiz Marcelo Sá Malbouisson, João Manuel da Silva Júnior and Maria José Carvalho Carmona \\ Department of Anesthesiology, Hospital das Clinicas, Faculdade de Medicina, University of São Paulo, Brazil
}

\begin{abstract}
Background: Thrombocytopenia is one of the most common laboratory abnormalities in intensive care unit patients (ICU), and seems to be related to ICU length of stay and mortality increasing. The purpose of this investigation was to evaluate the prevalence of thrombocytopenia in critically ill patients admitted to postoperative ICU with SIRS, sepsis and septic shock and its correlation with mortality.

Methods: a retrospective cohort study which analyzed data collected from patients admitted to a postoperative ICU was conducted over a period of 12 months. Patients were divided in two groups: thrombocytopenic patients ( $\mathrm{P}$ group) and non thrombocytopenic (C group). Patients were evaluated for 7 consecutive days using the SOFA score or even their discharge of the ICU, if this occurred before the day 7. Statistical analysis was performed using the Sigma Stat for Windows version 2.03 (SPSS Inc.). Results: Fifty-four patients with SIRS, sepsis and septic shock were included during the study period, 31 in P group and 23 in C. The prevalence of thrombocytopenia in critically ill patients in our study was $57 \%$, with $45 \%$ of mortality in $\mathrm{P}$ group against $13 \%$ of mortality in $\mathrm{C}$ group. The relative risk of death for critically ill patients who developed thrombocytopenia during hospitalization was 3.46 with a confidence interval ( $95 \% \mathrm{CI}) 3.17$ to 3.77 ( $\mathrm{p}=0.012$ ).
\end{abstract}

Conclusions: Critically ill patients that developed thrombocytopenia had risk of death 3.46 times higher than patients who did not develop thrombocytopenia during their stay in the ICU.

\section{Introduction}

Thrombocytopenia is one of the most common laboratory abnormalities in intensive care unit patients (ICU). The incidence of thrombocytopenia varies from $15 \%$ to $60 \%$, depending on the definition used and population evaluated [1]. A platelet count less than 100.000/ $\mathrm{mm}$ [2] is seen in another $30-50 \%$ of critically patients [2]. The possible mechanisms leading to thrombocytopenia in critically ill patients could involve the presence of disseminated intravascular coagulation (DIC), sepsis, massive hemorrage, thrombocytopenia induced by heparin or other drugs [3].

Several authors have been investigating thrombocytopenia as a prognostic marker of critically ill patients [1,3-5]. Some of these authors believe that thrombocytopenia is related to ICU length of stay and mortality increasing, as an independent factor, leading to an increasing of death risk of more than 4 times [3]. The progressive decrease in platelet count is also associated with a worse prognosis [4], as well as platelet count above 150,000 appears to be a predictor of good outcome [5].

The systemic inflammatory response syndrome (SIRS) and sepsis are among the leading causes of hospitalization and ICU mortality, as are the leading cause of thrombocytopenia in critically ill patients [2], so it's of great importance to establish which of these patients is more likely to have an unfavorable outcome. Thrombocytopenia can be a good prognostic marker for this group of patients [5], because the predisposition to complications such as bleeding, as well as a marker of multiple organ failure.

\section{Objective}

The study aimed to evaluate the prevalence of thrombocytopenia in critically ill patients admitted to postoperative ICU with SIRS, sepsis and septic shock and its correlation with mortality of these patients.

\section{Methods}

After approval by the Research Assessment Protocols Hospital das Clínicas da FMUSP (635/02 and SE/MA1/249/03), we conducted a retrospective cohort study which analyzed data collected from patients admitted to the postoperative ICU of Hospital das Clinicas, FMUSP, diagnosed with SIRS, sepsis and septic shock, over a period of 12 months. We considered thrombocytopenic patients whose that presented at least 2 laboratory tests on different days with platelet count $<100,000 / \mathrm{mm}^{3}$, during the ICU length of stay [5]. The severity of the patient's condition was assessed by APACHE II score (Acute Physiology and Chronic Health Evaluation II) [6] on the day of ICU admission. Patients were evaluated for 7 consecutive days using the SOFA (Sequential Organ Failure Assessment) [7-9], or even their discharge of the ICU, if this occurred before the day 7.

The diagnosis of SIRS was based in the presence of at least 2 of the following criteria: fever (temperature $>38.3^{\circ} \mathrm{C}$ ) or hypothermia (temperature $\left.<36^{\circ} \mathrm{C}\right)$, tachycardia $(>90 \mathrm{bpm})$, tachypnea $(>20 \mathrm{rpm})$ or hyperventilation $\left(\mathrm{PaCO}_{2}<32 \mathrm{mmHg}\right)$ and change in the number of leukocytes $(>12,000$ cells $/ 1$ or $<4,000$ cells/ $\mu$ l) or more than $10 \%$

Correspondence to: Domingos Dias Cicarelli, Department of Anesthesiology, Hospital das Clinicas, Faculdade de Medicina, São Paulo University, Brazil; E-mail: dcicarelli@uol.com.br

Key words: thrombocytopenia, intensive care unit, mortality

Received: March 04, 2015; Accepted: March 22, 2015; Published: March 24 2015 
of neutrophils [10,11]. Sepsis was diagnosed in cases of SIRS plus a suspected or confirmed infectious focus. The diagnosis of septic shock was made in patients with sepsis and refractory hypotension despite volume replacement, requiring the use of vasopressors $[10,11]$.

The study excluded patients who were suspected of heparin-induced thrombocytopenia or other medications and those with hematologic disorders that cause thrombocytopenia, patients on chemotherapy and that submitted to surgery with extracorporeal circulation.

Statistical analysis was performed using the Sigma Stat for Windows version 2.03 (SPSS Inc.). For continuous variables, were used the Student $t$ test and Mann-Whitney U-test and ANOVA for nonparametric variables of outcome. The relative risk of death with confidence interval was calculated between groups.

\section{Results}

Fifty-four patients with SIRS, sepsis and septic shock were included during the study period. These patients were divided into 2 groups: $\mathrm{P}$ group consisting of 31 patients who met the criteria for thrombocytopenia during their stay in the ICU and C group consisting of 23 patients without thrombocytopenia during the ICU length of stay. There were no significant differences in age, weight and gender between groups. The APACHE II score did not show statistically significant difference between groups. Demographic data of the study population was showed in Table 1 .

$\mathrm{P}$ group included 31 thrombocytopenic patients with 14 deaths, accounting for $45 \%$ of mortality. In the $\mathrm{C}$ group composed of 23 patients without thrombocytopenia, 3 patients died, accounting for $13 \%$ of mortality (Table 2). Thus, the relative risk of death for critically ill patients who developed thrombocytopenia during hospitalization was 3.46 with a confidence interval $(95 \% \mathrm{CI}) 3.17$ to 3.77 ( $\mathrm{p}=0.012$ ).

The prevalence of thrombocytopenia in critically ill patients in our study was $57 \%, 31$ patients in 54 thrombocytopenic patients.

Graph 1 shows the evolution of platelets count in both groups.

Graph 2 shows the evolution of the SOFA score of organ failure of the groups $\mathrm{P}$ and $\mathrm{C}$.

\section{Discussion}

We observed that patients with thrombocytopenia had risk of death

Table 1. Demographic data of groups.

\begin{tabular}{|c|c|c|c|}
\hline Demographic data & P group & C group & p \\
\hline Number of patients & 31 & 23 & - \\
\hline Age in years (SD \pm mean) & $61 \pm 19$ & $55 \pm 16$ & $0.18(\mathrm{~ns})$ \\
\hline Weight in kilograms (SD \pm mean) & $66,3 \pm 12,2$ & $69 \pm 11,6$ & $0.41(\mathrm{~ns})$ \\
\hline Gender (M/F) & $61 \% / 39 \%$ & $48 \% / 52 \%$ & $0.14(\mathrm{~ns})$ \\
\hline APACHE II & $18 \pm 5$ & $17 \pm 5$ & $0.45(\mathrm{~ns})$ \\
\hline Sepsis/septic shock & 16 & 11 & $0.62(\mathrm{~ns})$ \\
\hline
\end{tabular}

P group: thrombocytopenic patients; C group: non thrombocytopenic patients; SD: standard deviation; $\mathrm{M} / \mathrm{F}$ : male/female; ns: not statistically significant

Table 2. Relative risk of death in thrombocytopenic patients during ICU stay.

\begin{tabular}{|c|c|c|c|}
\hline Group & Survivors & Deaths & Total \\
\hline P & 17 & 14 & 31 \\
\hline C & 20 & 3 & 23 \\
\hline Total & 37 & 17 & 54 \\
\hline
\end{tabular}

P group: thrombocytopenic patients; $\mathrm{C}$ group: non thrombocytopenic patients
Evolution of platelets count of groups during ICU stay

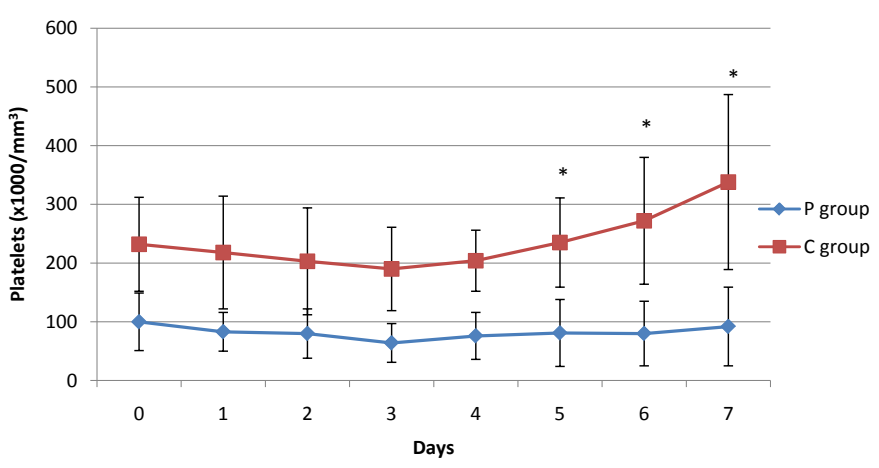

Graph 1. Evolution of platelets count of groups during ICU stay. Where * means $\mathrm{p}<0.001$

\section{SOFA score evolution}

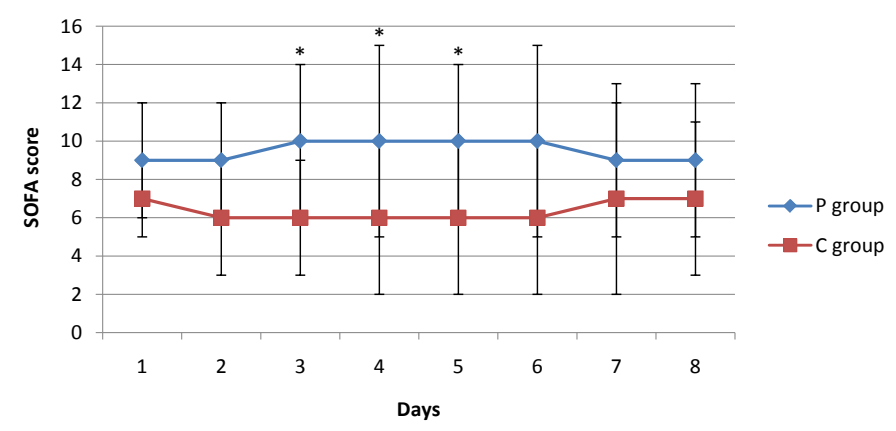

Graph 2. Evolution of SOFA score in groups during ICU stay.

Where $*$ means $\mathrm{p}<0.001$

3.46 times higher than patients who did not develop thrombocytopenia during their stay in the ICU. This finding is consistent with the literature [2].

Platelets play a key role in hemostasis and can be used as a marker of disseminated intravascular coagulation, where a significant decrease in platelet count should be interpreted as a deterioration signal in critically ill patients, especially in patients with sepsis or septic shock [4]. Sepsis is a triggering factor of thrombocytopenia, modifying platelets production, increasing their consumption, sequestration or destruction by the spleen or along the surface of endothelial $[2,4]$. The decreased of platelet production in septic patients, although possible, would be inconsistent in patients with elevated levels of pro-inflammatory cytokines that stimulate platelet production [2]. However, an increase in the hemophagocytosis by megakaryocytes, monocytes and macrophages may occurs in sepsis patients, due to increased production of stimulating factor macrophage colonyforming (M-CSF) [2]. At the same time that the number of platelets decreases by increasing the destruction, thrombin production (which is an important activator of platelets) increases, which might lead to disseminated intravascular coagulation [2]. Thus, microthrombus would be formed and deposited in microcirculation, decreasing peripheral tissue perfusion and consuming more platelets and clotting factors [2]. The activation, consumption and platelet destruction may occur in endothelial surface as a result of extensive interaction of platelets with the endothelial cells that occurs in sepsis, which can extend for different vascular beds in different organs contributing to multiple organ dysfunctions seen in these patients [2]. 
In our study, we observed that thrombocytopenic patients had more deaths than non-thrombocytopenic patients (Table 2), presenting higher SOFA score values between the third and the sixth day (Graph 2 ). Thus, the evaluation of the SOFA score confirms our main result when indicates greater severity of thrombocytopenic patients. The lack of statistically significant difference between groups regarding to the SOFA score in the last two days of evaluation, can be explained by the fact that some thrombocytopenic patients died during ICU stay, and some non thrombocytopenic patients have been discharged from the ICU during the period of 7 days. It is important to note the homogeneity of the groups when they were admitted to the ICU, observing that the APACHE II score applied to patients was not different between groups, indicating the same degree of severity of groups evaluated.

The $31.5 \%$ of mortality rate observed in our study was slightly lower than the mortality reported in the international literature that ranges from $35 \%$ to $55 \%$ in septic patients and $38 \%$ in postoperative ICU patients with thrombocytopenia [4,5]. Our population was not composed exclusively of septic patients and this may be the reason of the lower mortality observed in our study. Likewise, the prevalence of thrombocytopenia in our study was $57 \%$, closely to the prevalence reported by other authors that can varies from $30 \%$ to $50 \%[2,5]$.

Our study has some limitations, mainly related to being retrospective, thereby allowing no kind of intervention. In addition to this fact, the number of patients studied was small despite the great homogeneity of the groups evaluated.

In conclusion, low platelet count is a marker of severity of critical patients and progression of the underlying disease, being directly related to the prognosis and mortality of patients in the postoperative ICU.

\section{References}

1. Elgohary TS, Zaghla HE, Azab AME (2011) Role of thrombocytopenia as an independent prognostic marker in the critically ill patients with multiorgan failure. Med J Cairo Univ 79: 1-9

2. Vanderschueren S, De Weerdt A, Malbrain M, Vankersschaever D, Frans E, et al. (2000) Thrombocytopenia and prognosis in intensive care. Crit Care Med 28: 18711876. [Crossref]

3. Levi M, Schultz M (2010) Coagulopathy and platelet disorders in critically ill patients Minerva Anestesiol 76: 851-859. [Crossref]

4. Boechat TD, Silveira MF, Faviere W, Macedo GL (2012) Thrombocitopenia in sepsis an important prognosis factor. Rev Bras Ter Intensiva 24: 35-42. [Crossref]

5. Stéphan F, Hollande J, Richard O, Cheffi A, Maier-Redelsperger M, et al. (1999) Thrombocytopenia in a surgical ICU. Chest 115: 1363-1370. [Crossref]

6. Cook R, Cook D, Tilley J, Lee K, Marshall J; Canadian Critical Care Trials Group (2001) Multiple organ dysfunction: baseline and serial component scores. Crit Care Med 29: 2046-2050. [Crossref]

7. Vincent JL, Moreno R, Takala J (1996) The SOFA (sepsis-related organ failure assessment) score to describe organ dysfunction/failure. On behalf of the Working Group on Sepsis-Related Problems of the European Society of Intensive Care Medicine. Intensive Care Med 22: 707-710. [Crossref]

8. Vincent JL, De Mendonça A, Cantraine F (1998) Use of the SOFA score to assess the incidence of organ dysfunction/failure in intensive care units: results of a multicenter prospective study. Working group on "sepsis-related problems" of the European Society on Intensive Care Medicine. Crit Care Med 26: 1793-1800. [Crossref]

9. Pettilä V, Pettilä M, Sarna S, Voutilainen P, Takkunen O (2002) Comparison of multiple organ dysfunction scores in the prediction of hospital mortality in the critically ill. Crit Care Med 30: 1705-1711. [Crossref]

10. Dellinger RP, Levy MM, Carlet JM, Bion J (2008) Surviving sepsis campaign: International guidelines for management of severe sepsis and septic shock. Crit Care Med 36: 296-327. [Crossref]

11. Dellinger RP, Levy MM, Rhodes A, Annane E (2012) Surviving sepsis campaign: International guidelines for management of severe sepsis and septic shock. Crit Care Med 41: 580-637. [Crossref]

Copyright: (C2015 Cicarelli DD. This is an open-access article distributed under the terms of the Creative Commons Attribution License, which permits unrestricted use, distribution, and reproduction in any medium, provided the original author and source are credited. 